\title{
BMJ Open Development of a research platform for children with arthrogryposis multiplex congenita: study protocol for a pilot registry
}

Noémi Dahan-Oliel, ${ }^{1,2}$ Tanya Bedard, ${ }^{3}$ Vasiliki Betty Darsaklis, ${ }^{1}$ Judith Goslin Hall, ${ }^{4,5}$ Harold J P van Bosse, ${ }^{6}$ Reggie C Hamdy ${ }^{1,7}$

To cite: Dahan-Oliel N, Bedard T, Darsaklis VB, et al. Development of a research platform for children with arthrogryposis multiplex congenita: study protocol for a pilot registry. BMJ Open 2018;8:e21377. doi:10.1136/ bmjopen-2017-021377

- Prepublication history for this paper is available online. To view these files, please visit the journal online (http://dx.doi. org/10.1136/bmjopen-2017021377).

Received 11 January 2018 Revised 4 May 2018 Accepted 17 May 2018
Check for updates

For numbered affiliations see end of article.

Correspondence to Dr Noémi Dahan-Oliel; ndahan@shrinenet.org

\section{ABSTRACT}

Introduction Arthrogryposis multiplex congenita (AMC) describes a heterogeneous group of conditions with multiple congenital contractures. These conditions may be attributed to genetic or other factors inducing decreased fetal movements, including maternal and paternal factors. Discovering the underlying genetic pathways has important repercussions for prevention, gene therapy and genetic counselling. The current literature mainly consists of small-scale, single-site studies, limiting comparability and pooling of findings across individual studies. A pilot registry for children presenting with AMC is proposed to provide the framework for a large-scale AMC registry. This registry will provide the platform to support high-quality studies to inform the distribution, clinical practice and genetics contributing to this group of conditions. Methods and analysis The registry will be piloted on 40 families of children from birth to 21 years of age presenting with AMC. Data will be collected on the child (demographic and newborn variables), mother and father (demographic, lifestyle habits and medical history). To promote standardised data collection, a manual of operations will be developed. Descriptive statistics will be used to summarise relevant data, regression analyses will be used to explore associations to generate hypotheses regarding factors contributing to AMC. Qualitative analysis will also be used to better describe the various phenotypes.

Ethics and dissemination Ethics approval was obtained at the participating sites. The pilot registry will provide the platform for multisite AMC registry that will generate multiple research avenues to enhance current care and establish new therapies. Following this pilot study, the participant selection criteria will be refined and datasets will be expanded to include rehabilitation and surgical interventions, and genetic sequencing. The best timing for the questionnaire administration and frequency of followup prior to the implementation of a multisite AMC registry will be determined.

\section{BACKGROUND AND RATIONALE}

Arthrogryposis and arthrogryposis multiplex congenita (AMC) are descriptive terms, rather than specific diagnoses, used for a
Strengths and limitations of this study

- An international multidisciplinary team of experts contributed to the development of this registry.

- This is the first registry of its kind for arthrogryposis multiplex congenita.

- Sustainability of any registry is dependent on funding.

- This registry will provide a platform to generate future studies on the distribution, causes, interventions and outcomes.

heterogeneous group of conditions with multiple congenital contractures and are often used interchangeably. This group of conditions affects 1 in 3000 live births ${ }^{12}$ and results from a lack of fetal movement. ${ }^{3}$ Individuals born with these conditions exhibit multiple joint contractures in at least two different body areas, including the upper and lower limbs, spine and jaw ${ }^{1}$ as depicted in figures 1-3. AMC may represent complex cases as impairments in other systems including gastrointestinal, genitourinary and central nervous systems are common. ${ }^{4}$

Hall $^{5}$ recommended classifying cases using the following categories to aid in a specific diagnosis in AMC: (1) limb involvement only, (2) limb with other system involvement and (3) neuromuscular involvement with central nervous system dysfunction or intellectual disability. A specific diagnosis is based on the evaluation of pregnancy and delivery history, a detailed physical examination with documentation as to which joints are affected and the degree of extension and/ or flexion, the examination of radiographs and photographs, and the natural history of complications in response to different interventions. ${ }^{5}$ Laboratory tests, such as muscle biopsies, blood tests and genetic analysis, also contribute to a specific diagnosis. 


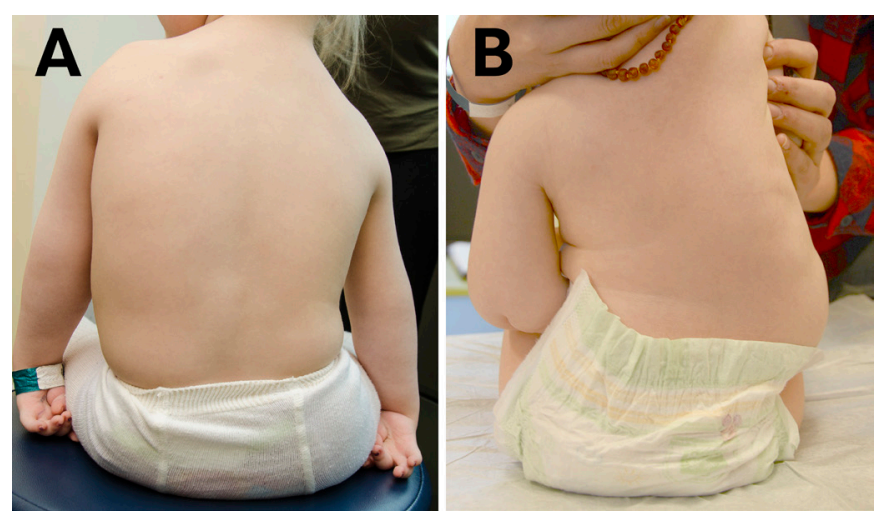

Figure 1 Spinal deformities of varying severity.

AMC may occur as part of inherited single-gene disorders or may occur sporadically. Currently, over 300 genes have been associated with $\mathrm{AMC}^{6}{ }^{7}$; however, it is unclear how these mutations lead to the clinical presentation of AMC as less than half of the patients have a mutation in one of the known AMC-related genes. ${ }^{8}$ Discovering the genetic pathways responsible for these conditions have important repercussions for prevention, gene therapy and genetic counselling. AMC may also be attributed to other factors inducing decreased fetal movements. These include maternal factors such as structural uterine anomalies that result in fetal crowding, exposure to teratogens, maternal conditions (such as myasthenia gravis and multiple sclerosis) and physical health status at the time of conception and during gestation. Paternal influences may also play a key role and need to be studied further. ${ }^{9}{ }^{10}$ Factors integral to understanding the long-term needs of individuals with rare conditions, such as individuals presenting with AMC, relate to the distribution and natural history and are lacking in the literature. The terminology and definitions used by various disciplines to classify and describe this group of conditions and applied outcome measures are often inconsistent. The International Classification of Diseases (ICD), a standard diagnostic tool used in epidemiology, and health management do not provide the
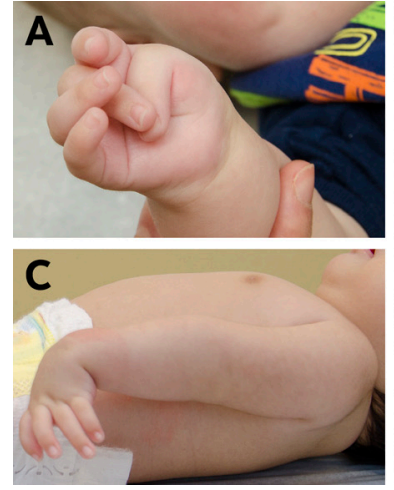

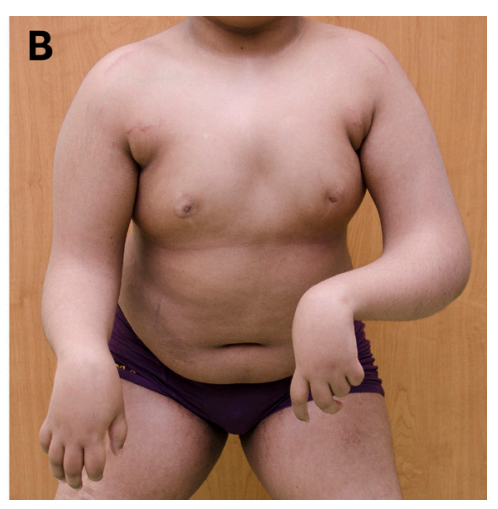

Figure 2 Images depicting upper limb involvement: (A) thumb-in-palm and overlapping fingers; (B) flexion contracture at the elbows and wrists, and absence of skin creases at the elbow; $(\mathrm{C})$ internal rotation with extension contracture at the elbow.
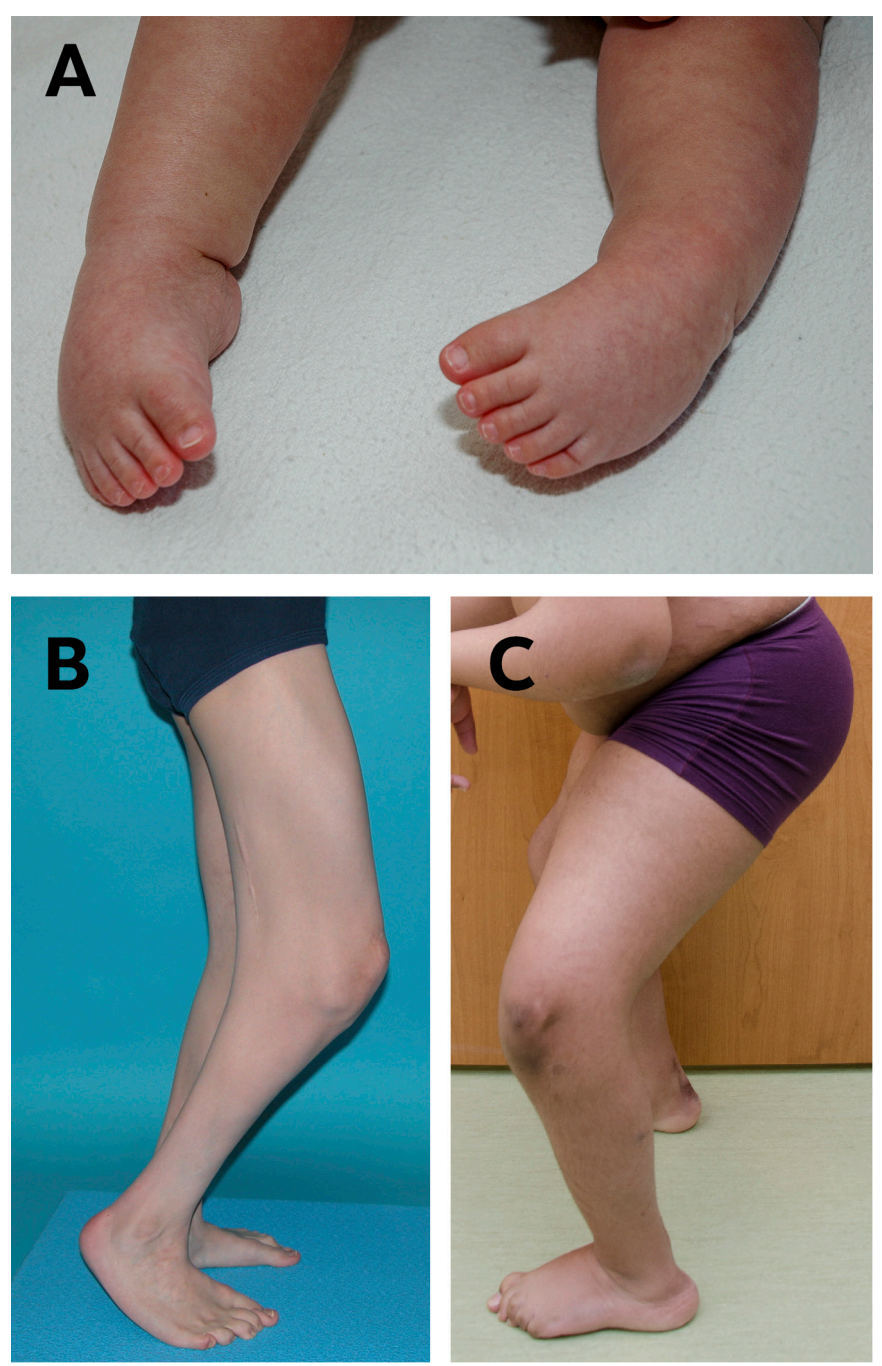

Figure 3 Images depicting lower limb involvement: (A) clubfeet, (B) flexion contracture of the knee and webbing in the popliteal region, $(\mathrm{C})$ flexion contracture at the hips.

necessary details to easily retrieve cases presenting with AMC for research and clinical purposes, nor does it indicate severity or aetiological information.

Research studies in AMC are often fragmented, isolated and are small scale. Conducting research in rare diseases poses several challenges. Investigator-initiated research often results in small sample sizes, ${ }^{11}$ precluding the use of robust statistical methods and limiting the findings' external validity. Comparability and pooling of findings across individual studies in rare diseases often lack standardisation in methodology and will vary in data collection, selection criteria, classification and outcome measures. ${ }^{12}$ Currently, there is no comprehensive registry or classification system to support the high-quality studies needed to inform the distribution, clinical practice and genetics contributing to this group of conditions. Multisite collaborations across a breadth of disciplines offer enormous scientific leveraging opportunities. The creation and implementation of a multisite AMC registry will provide the opportunity to collaborate and develop standardised methodologies to contribute to rigorous 
research and enhance current care and establish new therapies. Prior to the full implementation of the AMC registry, a pilot registry will be developed.

\section{Case definition}

A clear and standardised case definition is necessary to ensure that all potential participants of the registry can be systematically and correctly identified and approached for recruitment. Considering the heterogeneity of the population presenting with AMC, as well as the lack of proper coding systems within healthcare institutions to properly denote individuals with a clinical presentation of AMC, ${ }^{2}$ a specific case definition was developed through consultation with a panel of experts on AMC and registry development. This definition includes a description of clinical presentation, onset, factors associated with these conditions, and functional and long-term implications.

\section{Data elements}

The final dataset for the pilot registry was validated through the method proposed by Lawshe. ${ }^{13} 14$ Content validation of any instrument is crucial prior to piloting its use to ensure that an instrument measures all elements of the content but is free of unwanted or irrelevant details for the overall purpose ${ }^{15}$ Validation of the dataset is essential to ensure that the information collected will be pertinent to ultimate end users of the knowledge (clinicians, families, patients). The final dataset only includes items with high content validity ratios, thereby increasing the efficiency and significance of the data elements for the AMC registry. The specifics of this method and the results of the exercise will be shared through future publications.

The objectives of the pilot registry for AMC are to:

- Provide the framework to fully implement the AMC registry.

- Provide opportunities to refine participant selection criteria, and expand datasets to include rehabilitation, surgical management and genetic sequencing.

- Determine the best timing of questionnaire administration and frequency of follow-up.

- Resolve potential challenges before the full implementation of the AMC registry.

\section{METHODS}

\section{Study design}

The pilot registry is a cross-sectional multicentre registry. Retrospective data from the time of pregnancy, birth and neonatal period of the registered child will be collected for the pilot phase of this registry. The full AMC registry will consist of a prospective registry using both retrospective and cross-sectional data. This registry was initiated by a panel of experts which identified the gaps in research and clinical therapies for children presenting with AMC. Included disciplines were: rehabilitation, genetics, orthopaedics, clinical research and registry management. Additionally, patient representativeness was ensured through the inclusion of a patient advocate. Prior to full registry implementation, the registry will be pilot tested with 40

\section{PILOT PHASE}
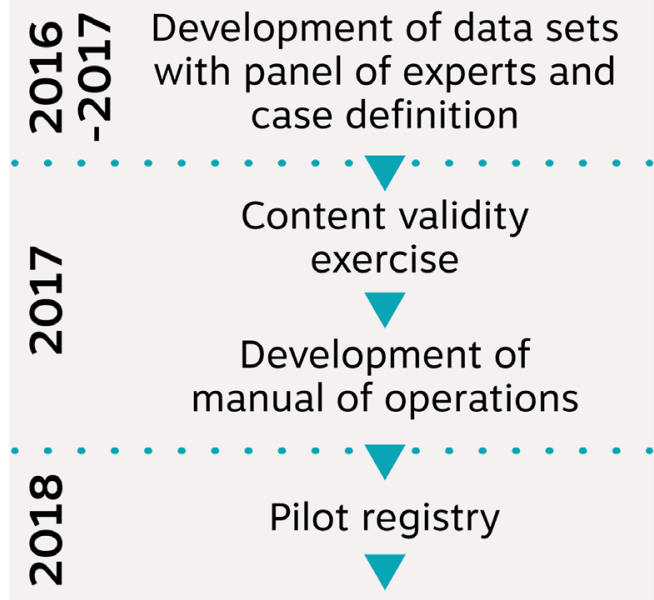

FUTURE DIRECTIONS
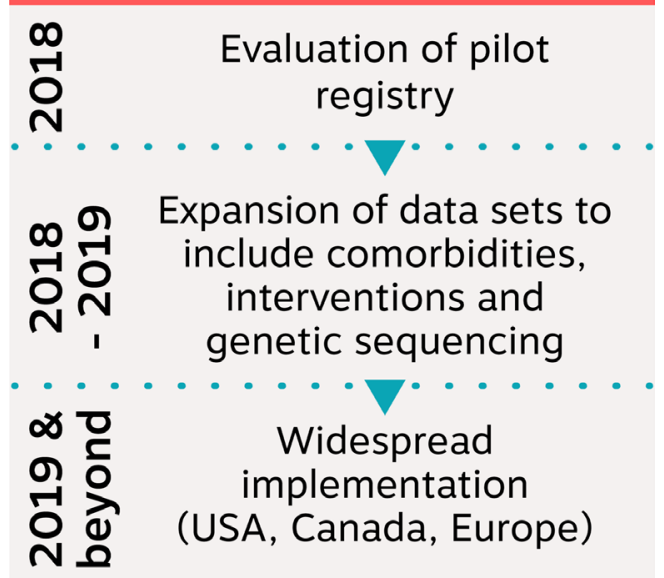

Figure 4 Timeline for the pilot registry and its projection.

families. Pilot testing is crucial to determine the feasibility of a study prior to launching a large-scale multisite project. It allows the investigators to solve potential issues prior to full expansion. Figure 4 represents the timeline for the pilot registry and its projection.

\section{Patient and public involvement}

Patient engagement has been intrinsic to the conception of the AMC registry. The initial panel of experts included the founder from a North American patient support group so that the youth and families' voices were considered from the start. During a knowledge-exchange day geared towards families and individuals with AMC and the patient-support group's annual meeting, it was identified that learning more about the genetics behind AMC, treatment effectiveness and long-term outcomes was a main priority. These issues are close to the hearts of those affected by AMC and are therefore integral to the registry. Additionally, content validation for the final data-set included patient perspectives to ensure that their concerns were being addressed. Results of the pilot phase 
of the registry will be shared with families and youth with AMC via newsletters through accessible channels such as the website for the support group.

\section{Study setting}

The AMC registry will be pilot tested at two Shriners Hospital for Children (SHC) sites: the SHC-Canada in Montreal, Quebec and SHC-Philadelphia. These two sites were purposefully selected as they have a relatively large, diverse population of patients presenting with AMC, thus facilitating recruitment and providing the opportunity to explore contributory factors of these conditions for the pilot phase.

\section{Eligibility criteria, sample size and recruitment procedures}

Families will be recruited based on having at least one child (0-21 years old) with the common clinical manifestation of multiple congenital contractures. Eligibility for the registry will be confirmed by the orthopaedic surgeons who are treating at both sites (RH and HVB). Participants will be recruited during their outpatient clinic visit at each respective site by the research assistant, who will provide information regarding the pilot registry and review the consent/assent forms as appropriate. A tentative appointment for the telephone interview will be scheduled. However, if preferred by the family, they will be contacted again by email to schedule an appointment. The primary caregiver from 40 families, 20 from each site, will be required to complete the telephone interview with the research assistant. Some attrition is expected (completed consent but incomplete interview); however, as this registry is a pilot, the attrition rate cannot yet be estimated and will be calculated after the completion of this pilot study. Recruitment will therefore continue until all 40 interviews have been completed. Total sample size and attrition rate will then be calculated and will be used to inform recruitment for the full multisite AMC registry.

\section{Data collection methods}

The AMC pilot registry was modelled after the established Canadian Cerebral Palsy registry and tailored using the reference manual 'Arthrogryposis: A Text Atlas'. ${ }^{16}$ This resulted in a manual of operations for a standardised data collection process and a comprehensive AMC-specific case report form (CRF) which includes the data elements for the pilot registry. These elements are listed in figure 5 and correspond to the child (eg, date of birth, place of birth, birth weight and gestation, race and ethnicity, diagnoses), maternal and paternal demographics (date of birth, residence, ethnicity, lifestyle habits), pregnancy (antenatal conditions), and birth (delivery information), maternal and paternal medical history (pre-existing conditions, prescription use).

Prior to piloting, a standardised training module will be completed by each research assistant to ensure that recruitment, data collection and data entry are performed in a consistent manner across both recruiting

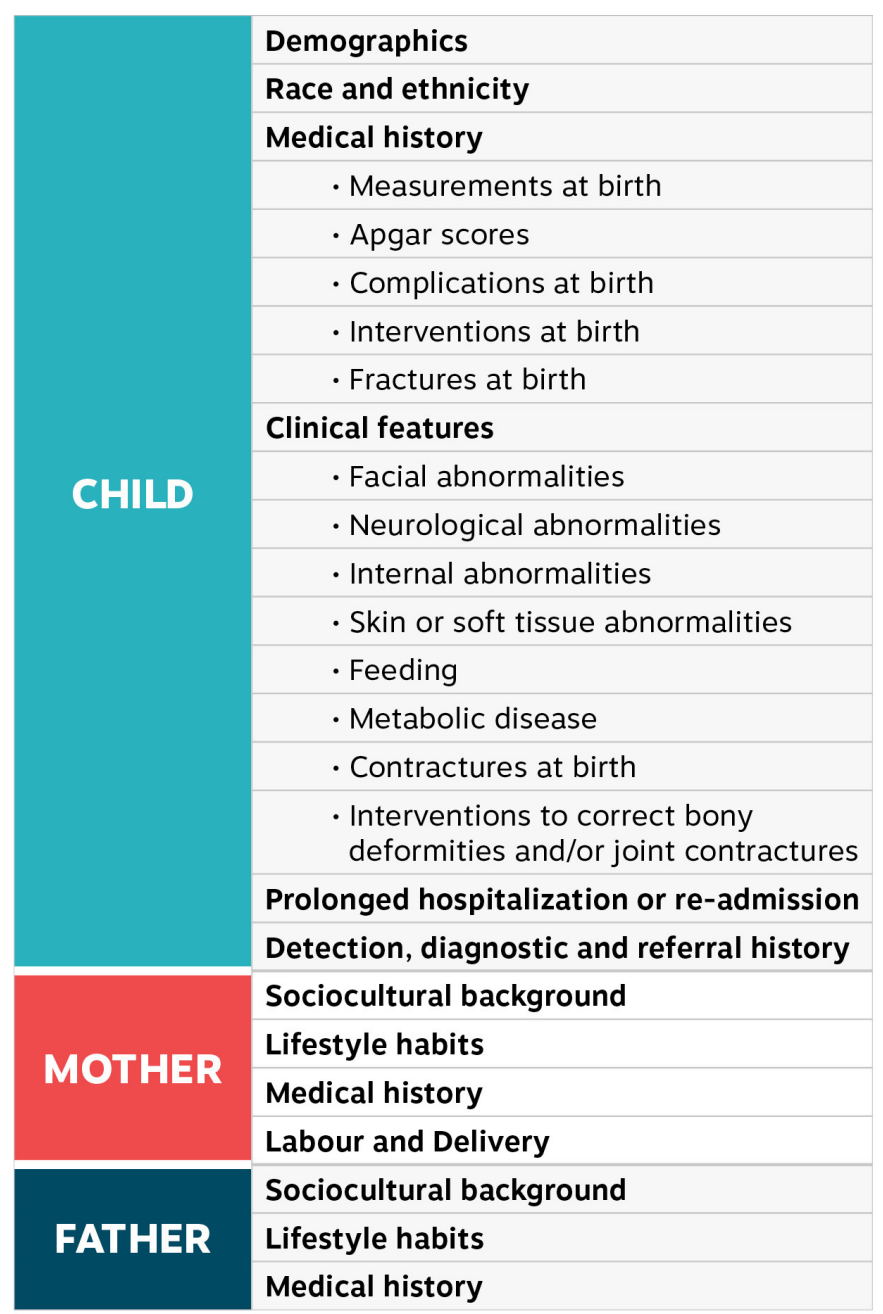

Figure 5 List of datasets for the pilot registry.

sites. Data will be collected from both the medical chart and the telephone interview, as using multiple sources to confirm the same data can minimise inadequacies found in one-source data. This data will be used to complete the CRF.

\section{Data management}

The CRF will be used to populate OnCore, a secure electronic database used by the Shriners system. This common method of data entry facilitates easy access to the comprehensive inventory of information and enables sharing between facilities on secured servers. ${ }^{17}$ A data-monitoring committee is not assigned to this pilot registry as no adverse events are expected since no interventions will be administered to participants as part of this pilot registry. Data will be secured and password protected and only accessed by registry personnel at the Shriners Hospitals for Children.

Essential to standardised data collection will be the manual of operations which will include detailed data collection procedures, a data dictionary, documentation requirements, validation rules and enhanced ICD-10 codes to ensure straightforward data retrieval. 


\section{Statistical analysis}

Descriptive statistics will be used to summarise relevant data. These will be reported as means, medians and proportions with corresponding measures of error as deemed appropriate. Regression analyses will be used to explore associations to generate hypotheses regarding risk factors, interventions and outcome which will be further explored in the multisite AMC registry. Qualitative analysis will also be used to describe the various phenotypes of AMC conditions. Additionally, the information collected from the two sources (ie, telephone interview and medical chart) will be checked for completeness. If a discrepancy between the two sources is identified, two researchers (ND-O and $\mathrm{TB}$ ) will select the data source to be used based on the variable collected. Additional sources of information often give more insight into a topic, and multiple sources provide verification and validity while complementing similar data. The data source (ie, telephone interview and medical chart) will therefore be determined a priori based on this comparison. ${ }^{18}$ By targeting medical chart requisitions, data collection will be facilitated in the larger scale implementation.

\section{Closure of pilot registry}

The pilot phase of the AMC registry will end once 40 families have completed the telephone interview. Recruitment procedures will be evaluated and preliminary data analyses will be completed. Expansions to the registry content and to participating sites will be reviewed with the panel of experts.

\section{ETHICS}

\section{Research ethics approval and protocol amendments}

Research ethics and administrative site approvals have been received at both the SHC-Canada and SHC-Philadelphia sites. Since the registry is not a clinical trial, this protocol has not been registered. Minor wording revisions were required to the consent and assent forms prior to obtaining final ethics approval. The protocol was accepted as submitted.

\section{Consent/assent}

Patient participation is completely voluntary, and informed written consent will be obtained prior to participation. This consent will be obtained while the patients are present for AMC clinic by the research assistant assigned to the study. Either or both parents/guardians will sign the consent form as they will be the ones to complete the questionnaire over the telephone. For the Canadian site, assent will be obtained for children between the ages of 7-14 years. For patients older than 14 years of age, consent will be obtained from both the parent and the child to ensure participation. In the US site, children between the ages of 7 and 17 years provide assent while those over 18 years of age provide consent. These procedures are state and province specific and are approved by the local research ethics boards.

\section{Confidentiality and access to data}

OnCore is used in all SHC studies for the management of clinical research data. This secure database implements industry best practices and ongoing monitoring activities, such as daily-encrypted backups of hosted customer data. Only authorised personnel will have access to the CRF which will be stored in a locked file cabinet. Specific individual information in the registry will not be shared with other persons, entities or companies unless obligated by legal authorities. Data collection will be performed locally and anonymised in the online OnCore Enterprise Research software.

\section{Dissemination policy}

Results from the pilot phase of the registry will be shared through a manuscript regarding the findings of this study. Additionally, information briefs will be provided to the participating clinicians and families. These information briefs will contain the most pertinent findings from the pilot phase, as well as future directions for research, specifically for the full multisite registry. A summary of the research findings will also be made available to the sponsor of the study. Authorship will be based on the following criteria: substantial contribution to the conception or design of the work; or the acquisition, analysis or interpretation of data.

\section{DISCUSSION}

The pilot AMC registry is designed to provide a platform for the generation of hypothesis-driven multicentre longitudinal studies in AMC. To date, little is known about these conditions with respect to aetiology and natural history. Evidence and effectiveness of various therapies (genetic, surgical and rehabilitative) are lacking. Considering the relative rarity and heterogeneity of AMC, large sample sizes to conduct research with generalisable outcomes is nearly impossible to gleam from single-site studies. Multisite collaboration is warranted and can be achieved through a registry. A standard case definition is essential to ensure that all potential participants are consistently recruited across sites so that the sample size can be as large as possible. A comprehensive CRF is essential to ascertain all salient information required for future research avenues. The creation of a manual of operations will facilitate the implementation of an AMC registry by providing standardisation regarding data-collection procedures, and definitions and enhanced ICD-10 codes to be used for cases with different types of AMC. The generation of an online database that can be shared between sites will facilitate data collection, data entry and analysis.

The novelty of this approach with this population requires a pilot phase of the AMC registry to test feasibility (including need for personnel training) and impact on families. Piloting the registry on 40 participants across two SHC sites will provide important information with respect to ease of recruitment, length of administration and ease of 
execution. Random audits on $20 \%$ of the data registry (four at each site) will contribute to data quality assurance.

\section{SUMMARY}

Establishing a pilot AMC registry will provide the platform for a full expansion of the AMC registry to generate multiple research avenues to enhance current care and establish new therapies. Following this pilot study, we will refine the participant selection criteria and determine the best timing for the questionnaire administration and frequency of follow-up. As AMC includes a group of rare conditions, this protocol will also serve to guide the establishment of future rare disease registries.

\section{Author affiliations}

${ }^{1}$ Shriners Hospitals for Children, Montreal, Quebec, Canada

${ }^{2}$ School of Physical and Occupational Therapy, McGill University, Montreal, Quebec, Canada

${ }^{3}$ Alberta Congenital Anomalies Surveillance System, Alberta Children's Hospital, Calgary, Alberta, Canada

${ }^{4}$ Department of Medical Genetics, University of British Columbia and BC Children's Hospital, Vancouver, British Columbia, Canada

${ }^{5}$ Department of Pediatrics, University of British Columbia and BC Children's Hospital, Vancouver, British Columbia, Canada

${ }^{6}$ Shriners Hospitals for Children, Philadelphia, Pennsylvania, USA

${ }^{7}$ Department of Pediatric Surgery, McGill University Health Centre The Montreal Children's Hospital, Montreal, Quebec, Canada

Acknowledgements The authors acknowledge the administrative support of Carla Evangeliste and Nicole Young, graphic and medical illustrations by Guylaine Bédard and Denis Alves, and the scientific, clinical and expert contributions of Dr Bonita Sawatzky, Ani Samargian, Kathleen Montpetit, Caroline Elfassy, Rita Yap, Emily Lecker, Dr Maryam Oskoui and Dr Michael Shevell. The authors would also like to thank the families and patients who participated in the various patient engagement activities and who contributed to the final dataset development.

Contributors ND-0, RCH, TB, HJPvB and JGH initiated and planned this study as well as developed all associated materials. ND-0, TB and VBD wrote the protocol and the paper. ND-0, RCH, TB, HJPvB and JGH obtained funding. All authors read and approved the final version of this paper. ND-0, RCH and HJPvB will conduct the pilot phase of the registry.

Funding The development of the datasets, content validity and pilot phases are funded by a Developmental Clinical Grant from the Shriners Hospitals for Children grant number 79150 . The planning activities and face-to-face meeting for the panel of experts were funded by a planning and dissemination grant from the Canadian Institutes of Health Research-Institute of Musculoskeletal Health and Arthritis grant number 144020.

Competing interests None declared.

Patient consent Parental/guardian consent obtained.

Ethics approval Administrative site approval was obtained from the Department of Medical Research at Shriners Hospitals for Children Sponsor (CAN1711). Research ethics approval was obtained from the McGill University Faculty of Medicine Institutional Review Board (A00-M34-17A) for SHC-Canada and from the Western Institutional Review Board (1182229) for SHC-Philadelphia.
Provenance and peer review Not commissioned; externally peer reviewed.

Open access This is an open access article distributed in accordance with the Creative Commons Attribution Non Commercial (CC BY-NC 4.0) license, which permits others to distribute, remix, adapt, build upon this work non-commercially, and license their derivative works on different terms, provided the original work is properly cited and the use is non-commercial. See: http://creativecommons.org/ licenses/by-nc/4.0/

(C) Article author(s) (or their employer(s) unless otherwise stated in the text of the article) 2018. All rights reserved. No commercial use is permitted unless otherwise expressly granted.

\section{REFERENCES}

1. Hall JG. Arthrogryposis multiplex congenita: etiology, genetics, classification, diagnostic approach, and general aspects. J Pediatr Orthop B 1997;6:159-66.

2. Lowry RB, Sibbald B, Bedard T, et al. Prevalence of multiple congenital contractures including arthrogryposis multiplex congenita in Alberta, Canada, and a strategy for classification and coding. Birth Defects Res A Clin Mol Teratol 2010;88:1057-61.

3. Bevan WP, Hall JG, Bamshad M, et al. Arthrogryposis multiplex congenita (amyoplasia): an orthopaedic perspective. J Pediatr Orthop 2007;27:594-600.

4. Bernstein RM. Arthrogryposis and amyoplasia. J Am Acad Orthop Surg 2002;10:417-24

5. Hall JG. Arthrogryposis (multiple congenital contractures): diagnostic approach to etiology, classification, genetics, and general principles. Eur J Med Genet 2014;57:464-72.

6. Hall JG. Genetic aspects of arthrogryposis. Clin Orthop Relat Res 1985;194:44-53.

7. Hunter JM, Kiefer J, Balak CD, et al. Review of X-linked syndromes with arthrogryposis or early contractures-aid to diagnosis and pathway identification. Am J Med Genet A 2015;167A:931-73.

8. Bamshad M, Van Heest AE, Pleasure D. Arthrogryposis: a review and update. J Bone Joint Surg Am 2009;91(Suppl 4):40-6.

9. Reddihough DS, Collins KJ. The epidemiology and causes of cerebral palsy. Aust J Physiother 2003;49:7-12.

10. Feinberg JI, Bakulski KM, Jaffe AE, et al. Paternal sperm DNA methylation associated with early signs of autism risk in an autismenriched cohort. Int J Epidemiol 2015;44:1199-210 https://doi.org/.

11. Augustine EF, Adams HR, Mink JW. Clinical trials in rare disease: challenges and opportunities. J Child Neurol 2013;28:1142-50.

12. Griggs RC, Batshaw M, Dunkle $M$, et al. Clinical research for rare disease: opportunities, challenges, and solutions. Mol Genet Metab 2009;96:20-6.

13. Lawshe $\mathrm{CH}$. A quantitative approach to content validity. Pers Psychol 1975;28:563-75.

14. Wilson FR, Pan W, Schumsky DA. Recalculation of the critical values for Lawshe's content validity ratio. Measurement and Evaluation in Counseling and Development 2012;45:197-210.

15. Portney LG, Watkins MP. Foundations of clinical research: applications to practice. 3rd edn. Upper Saddle River: NJ: Pearson \& Prentice Hall, 2009:892.

16. Staheli LT, Hall JG, Jaffe KM, et al. Arthrogryposis: A text atlas. 1st edn. New York: Cambridge University Press, 1998:158 pages.

17. Gliklich R, Dreyer N, Leavy M, eds. Registries for Evaluating Patient Outcomes: A User's Guide: In. Two volumes. (Prepared by the outcome DEcIDE Center [Outcome Sciences, Inc., a Quintiles company] under Contract No. 290200500351 TO7.) AHRQ Publication No. 13(14)-EHC111. 3rd edition. Rockville, MD: Agency for Healthcare Research and Quality, 2014. http://www. effectivehealthcare.ahrq.gov/registries-guide-3.cfm. (accessed 15 Dec 2017).

18. Mant J, Murphy M, Rose $\mathrm{P}$, et al. The accuracy of general practitioner records of smoking and alcohol use: comparison with patient questionnaires. J Public Health Med 2000;22:198-201. 